\title{
Metabolomics Approach to Studying Minimally Processed Peach (Prunus persica) Fruit
}

\author{
R. Tosetti, F. Martinelli and P. Tonutti \\ Scuola Superiore Sant'Anna \\ Piazza Martiri della Libertà 33 \\ 56127 Pisa \\ Italy
}

\author{
D.K. Barupal \\ Genome Center, University of California \\ One Shields avenue \\ Davis CA 95616 \\ USA
}

Keywords: fresh-cut produce, metabolites, peach genotypes, quality, wound ethylene

\begin{abstract}
Fresh-cut fruit products for both retail and food service applications have increasingly appeared in the market place in recent years. Among different fruit types, peaches can be used and are highly appreciated as fresh-cut product although their commercial success is limited due to their short shelf-life and the quick onset of wounding-related physiological reactions. In this work we analyzed the technological and physiological changes induced by fresh-cut preparation in three different types of peach cultivars: 'Fayette' (melting), 'Wilhelmina' (non-melting) and 'Ghiaccio3' (stony hard). We performed a metabolite targeting profiling to focus on the changes in organic acid levels, important components of fruit taste and organoleptic quality of peaches. Interestingly, 'Ghiaccio3' showed an increase of several organic acids after cutting while 'Fayette' and 'Wilhelmina' showed unchanged amounts or a general reduction. Cutting induces a similar pattern of change in important metabolites (i.e., dehydroascorbate, alanine) in all the three peach types while other metabolites (i.e., citric acid) appeared to be differentially regulated in the considered peach cultivars.
\end{abstract}

\section{INTRODUCTION}

Peach (Prunus persica L.) belongs to the Rosaceae family and originated in China. Currently, in the world the three major producing countries are China, Italy and the United States in the northern hemisphere and Chile, South Africa and Australia in the southern hemisphere (Lurie and Crisosto, 2005). Phenotypically, peach fruits can be classified according to different parameters including, for example, the flesh color and pulp texture. These aspects are particularly important considering fresh-cut preparation. The USDA and FDA define "fresh-" and "minimally-processed" as those fruits and vegetables that have been washed, freshly-cut, packaged and maintained with refrigeration. They remain in a fresh state, ready to eat or cook, without freezing, thermal processing, or treatments with additives or preservatives. Peach fruits can be commercialized as freshcut but they are limited to their short shelf-life, because of cut surface browning, a rapid loss of firmness and tissue breakdown (Gorny et al., 1998, 1999).

Based on the flesh softening process, peaches can be divided into melting or nonmelting type. Differences in the various softening behaviours during ripening stage are due to the polygalacturonase (PG) activity that diverges between these two peach types (Lester et al., 1996). Indeed, at post-harvest stage non-melting peaches maintain firmness due to the mutation in a polygalacturonase gene despite which they produce ethylene. This type of fruit has been typically directed to the processing sector although the interest for the fresh market has increased because of their peculiar texture. A third type of peach is the so-called stony hard; these fruits are characterized by a lack of ethylene production at ripening due to a single recessive gene and in consequence they keep high firmness values even at post-harvest stage (Haji et al., 2001, 2005).

In this study we used a metabolite profiling approach to compare changes in organic acids in minimally processed peaches (Prunus persica L.) belonging to three fruit typologies: melting (yellow flesh, 'Fayette'), non-melting (yellow flesh, 'Wilhelmina') and stony hard (white flesh, 'Ghiaccio3'). 


\section{MATERIALS AND METHODS}

We analysed, at phenotypic and metabolomic level, the fresh-cut products obtained from different normal acid peach cultivars: 'Fayette' (melting), 'Wilhelmina' (non-melting), 'Ghiaccio3' (stony hard). 'Fayette' is a commercial peach cultivar usually used for fresh market, it has medium-size fruits characterized by a rapid decrease of firmness during ripening stage. 'Wilhelmina' is characterized by a mutation in the ripening-related endo-polygalacturonase gene and by the lack of the melting process at ripening. Its fruits are often used in the food industry for canning. 'Ghiaccio3' is characterized by the lack of ethylene production and reduced firmness loss at ripening. For each cultivar, physiological and metabolomic changes were evaluated comparing uncut (control) and cut (minimally processed) fruits during three days of storage at $4{ }^{\circ} \mathrm{C}$. For both categories, 15 fruits were considered and a part was immediately analysed after cutting $\left(T_{0}\right)$, the other samples were monitored 24 hours $\left(T_{1}\right), 48$ hours $\left(T_{2}\right)$, and 72 hours $\left(\mathrm{T}_{3}\right)$ from cutting. Changes in colour were measured using a Minolta CM2500 and the hue $\left(\mathrm{h}_{\mathrm{ab}}\right)$ index has been considered. For ethylene production, samples were incubated for 1 hour at RT in appropriate holders, and $1 \mathrm{ml}$ of the incubation air was injected in a gas chromatograph (DANI 3200) as described by Tonutti et al. (1997). The flesh firmness was determined with a digital fruit penetrometer, probe of $8 \mathrm{~mm}$. For each analysis 3 biological replicates composed by a pool of 4-6 pieces were evaluated from both of the two categories. Metabolomic analyses were carried out on control and $\mathrm{T}_{1}$ samples $(24 \mathrm{~h}$ after cutting) using GC-MS-TOF Pegasus II. Six replicates (different fruits) were used for each of the two categories. Fruits were frozen in liquid nitrogen, and stored at $-80^{\circ} \mathrm{C}$ until analysis. For each sample, $20 \mathrm{mg}$ pulp was ground in $2 \mathrm{ml}$ pre-chilled extraction solvent (MetOH: $\left.\mathrm{CHCl}_{3}, 1: 1, \mathrm{v} / \mathrm{v}\right)$. After vortexing and centrifugation $\left(3000 \mathrm{rcf}\right.$ for $2 \mathrm{~min}$ at $4{ }^{\circ} \mathrm{C}$ ) the supernatant was aliquoted and analyzed with a Pegasus III TOF GC mass spectrometer. The relative concentrations were determined by peak area $\left(\mathrm{mm}^{2}\right)$. All peak detections were manually checked for false-positive and false-negative assignments. Data analysis was performed with Statistica software and using the Binbase database developed by Fiehn research group (UCDavis, CA, USA).

\section{RESULTS AND DISCUSSION}

Firmness analysis showed that 'Ghiaccio3' peaches maintained a high level of texture after cutting $\left(\mathrm{T}_{0}: 5.91-6.62 \mathrm{~kg} ; \mathrm{T}_{3}: 4.89-5.60 \mathrm{~kg}\right)$; while in 'Fayette' and 'Wilhelmina', texture rapidly decreased (data not shown). Considering color analysis (Fig. 1), yellow flesh peaches ('Fayette' and 'Wilhelmina') showed similar $h_{a b}$ index comparing cut and uncut fruits while in white flesh 'Ghiaccio3' $h_{a b}$ index changed from negative to positive values immediately after cutting. As expected, ethylene production in 'Ghiaccio3' peaches was absent during all of the post-harvest stage (Fig. 2). In 'Fayette' fruits, ethylene slightly increased at $T_{1}$ reaching values of $15 \mathrm{nl} / \mathrm{g} / \mathrm{h}$. Indeed, 'Wilhelmina' showed a slow increase of ethylene production until $\mathrm{T}_{1}$, followed by a rapid increase reaching the maximum level at $T_{2}$. At $T_{3}$, ethylene level was extremely low.

We performed a metabolite targeting profiling on these three different peach types focusing on organic acids. In ripe peaches, the main organic acids are malic and citric acids. Peach fruits can be grouped into two types according to their $\mathrm{pH}$ value: the normally-acid ( $\mathrm{pH}$ below 4.0) and the low-acid phenotypes ( $\mathrm{pH}$ above 4.0) (Dirlewanger et al., 1998). Organic acids are important components of fruit taste and have a strong impact on the overall organoleptic quality of peaches since fruit taste depends on the content and type of soluble sugars and organic acids (Pangborn, 1963). Interestingly, metabolite profiling showed that the amount of several organic acids was increased after cutting in 'Ghiaccio3' while it remained unaltered in the other two cultivars. Indeed, an increase for alpha-ketoglutaric acid, aspartic acid, fumaric acid, glyceric acid, lactic acid, palmitic acid, salicylic acid, stearic acid was only observed in 'Ghiaccio3' (Fig. 3A). On the other hand, citric acid, dehydroascorbate, isocitric acid increased in 'Wilhelmina' after cutting whereas other organic acids such as citric acid, phosphoric acid, malic acid decreased in 'Fayette'. Some metabolites (e.g., dehydroascorbate, alanine) showed a 
similar pattern of modulation after cutting in the three cultivars while others (e.g., citric acid) appeared differentially regulated (Fig. 3B).

In conclusion, results demonstrated that a metabolomic approach can be used to characterize the different metabolic changes in peach genotypes that can be used for fresh-cut preparation. Besides the difference in ethylene biosynthesis, several organic acids appeared to accumulate at $24 \mathrm{~h}$ after cutting in 'Ghiaccio3' and 'Wilhelmina' but not in 'Fayette'. These differences would affect organoleptic quality in the three different types of peaches. Whether these (and other) changes are the result of differences in ethylene production in wounded tissues remains to be elucidated. These results will be integrated with microarray and gene expression analyses for a systems biology approach that will allow us to gain insight into metabolic changes in fresh-cut products.

\section{ACKNOWLEDGEMENTS}

This work has been financially supported by the DRUPOMICS (Mipaf) project.

\section{Literature Cited}

Dirlewanger, E., Pronier, V., Parvery, C., Rothan, C., Guye, A. and Monet, R. 1998. Genetic linkage map of peach [Prunus persica (L.) Batsch] using morphological and molecular markers. Theor. Appl. Genet. 97:888-895.

Gorny, J.R., Hess-Pierce, B. and Kader, A.A. 1998. Effects of fruit ripeness and storage temperature on the deterioration rate of fresh-cut peach and nectarine slices. HortScience 33:110-113.

Gorny, J.R., Hess-Pierce, B. and Kader, A.A. 1999. Quality changes in fresh-cut peach and nectarine slices as affected by cultivar, storage atmosphere and chemical treatments. J. Food Sci. 64:429-432.

Haji, T., Yaegaki, H. and Yamaguchi, M. 2001. Changes in ethylene production and flesh firmness of melting, nonmelting and stony hard peaches after harvest. J. Jpn. Soc. Hort. Sci. 70:458-459.

Haji, T., Yaegaki, H. and Yamaguchi, M. 2005. Inheritance and expression of fruit texture melting, non-melting and stony hard in peach. Sci. Hort. 105:241-248.

Lester, D.R., Sherman, W.B. and Atwell, B.J. 1996. Endopolygalacturonase and the melting flesh (M) locus in peach. J. Amer. Soc. Hort Sci.121:231-235.

Lurie, S. and Crisosto, C.H. 2005. Chilling injury in peach and nectarine. Postharvest Biol. Tec. 37:195-208.

Pangborn, R.M. 1963. Relative taste intensities of selected sugars and organic acids. J. Food Sci. 28:726-733.

Tonutti, P., Bonghi, C., Ruperti, B., Tornielli, G.B. and Ramina, A. 1997. Ethylene evolution and 1-aminocyclopropane-1-carboxylate oxidase gene expression during early development and ripening of peach fruit. J. Am. Soc. Hort. Sci. 122:642-647. 


\section{Figures}

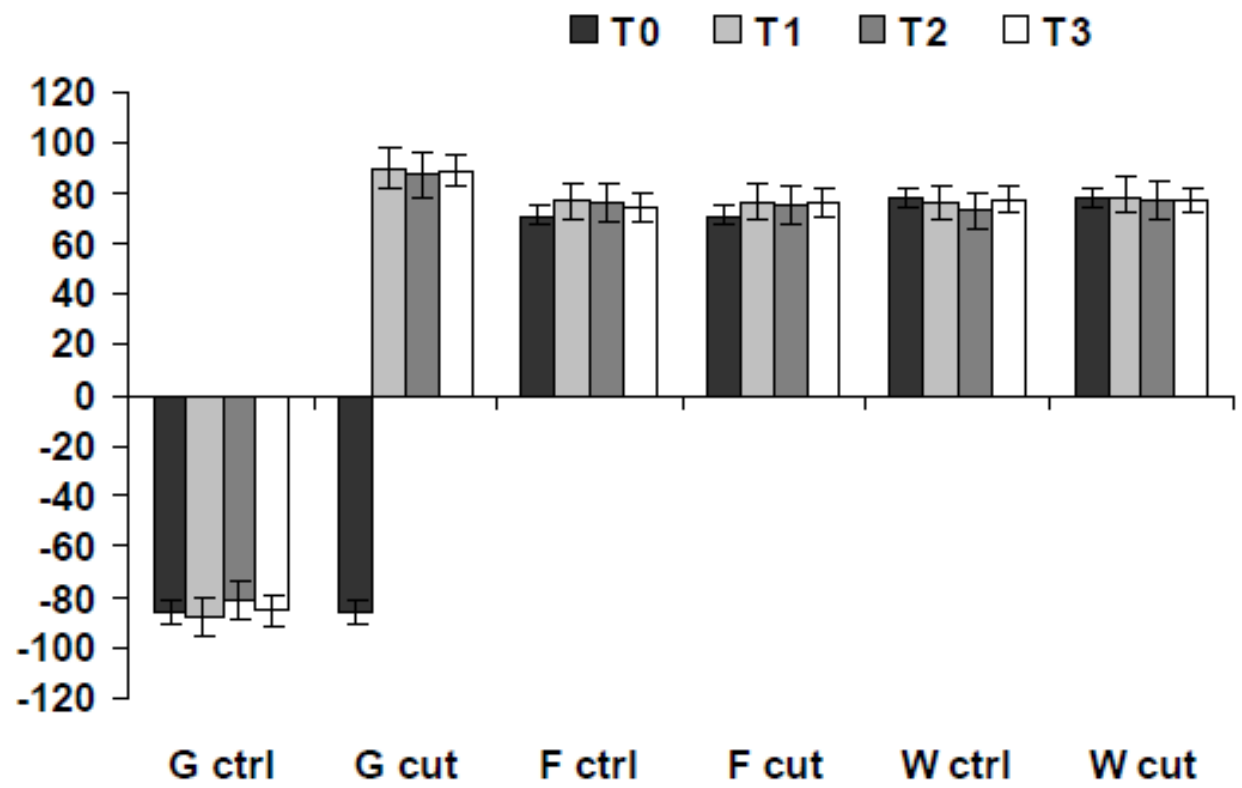

Fig. 1. Colorimetric analysis (hue index, $h_{a b}$ ) of 'Ghiaccio3', 'Fayette' and 'Wilhelmina' peaches after cutting compared to the same stage without cutting (control). Gctrl= 'Ghiaccio3' control, Gcut='Ghiaccio3' after cutting, Fctrl='Fayette' control, Fcut='Fayette' after cutting, Wctrl='Wilhelmina' control, Wcut='Wilhelmina' after cutting. $T_{0}, T_{1}, T_{2}$ and $T_{3}$ as described in Materials and Methods. Values are the mean $\pm \mathrm{SE}$ of 3 replicates.

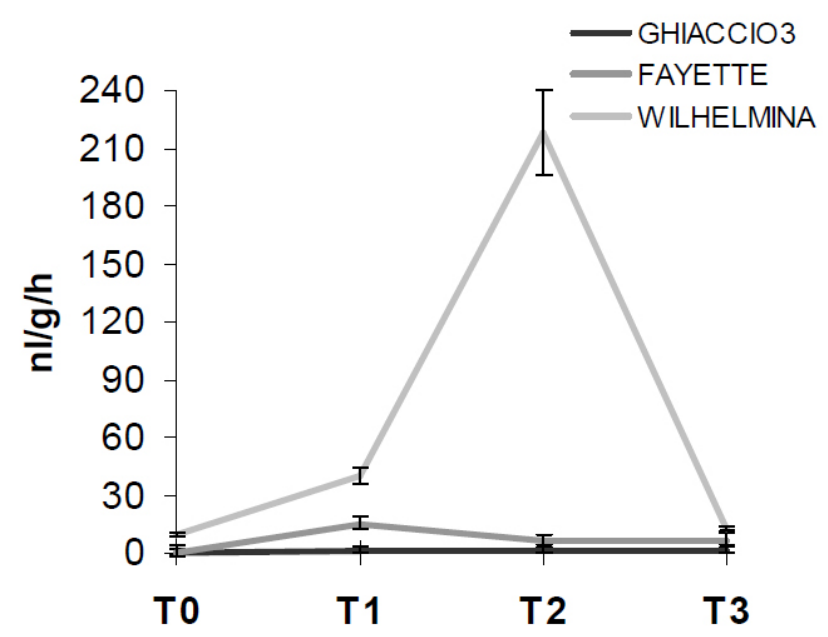

Fig. 2. Ethylene production in the different types of peach fruits at three stages after cutting: $\mathrm{T}_{0}, \mathrm{~T}_{1}, \mathrm{~T}_{2}$ and $\mathrm{T}_{3}$ as described in Materials and Methods. Values are the mean $\pm \mathrm{SE}$ of 3 replicates. 
A

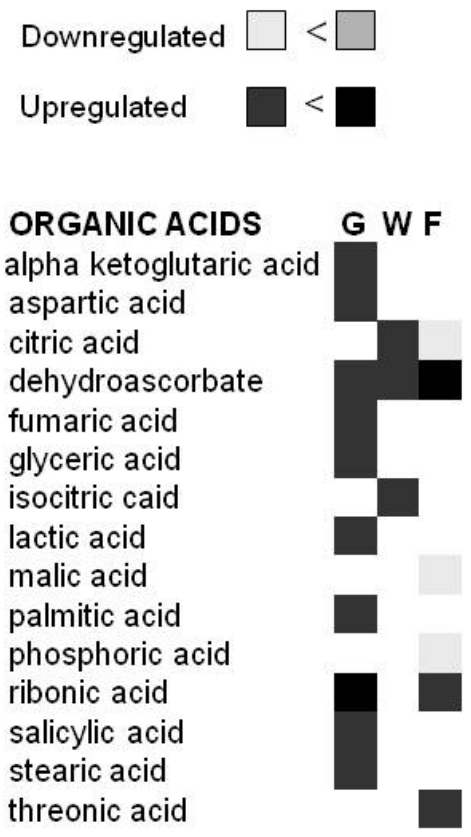

B
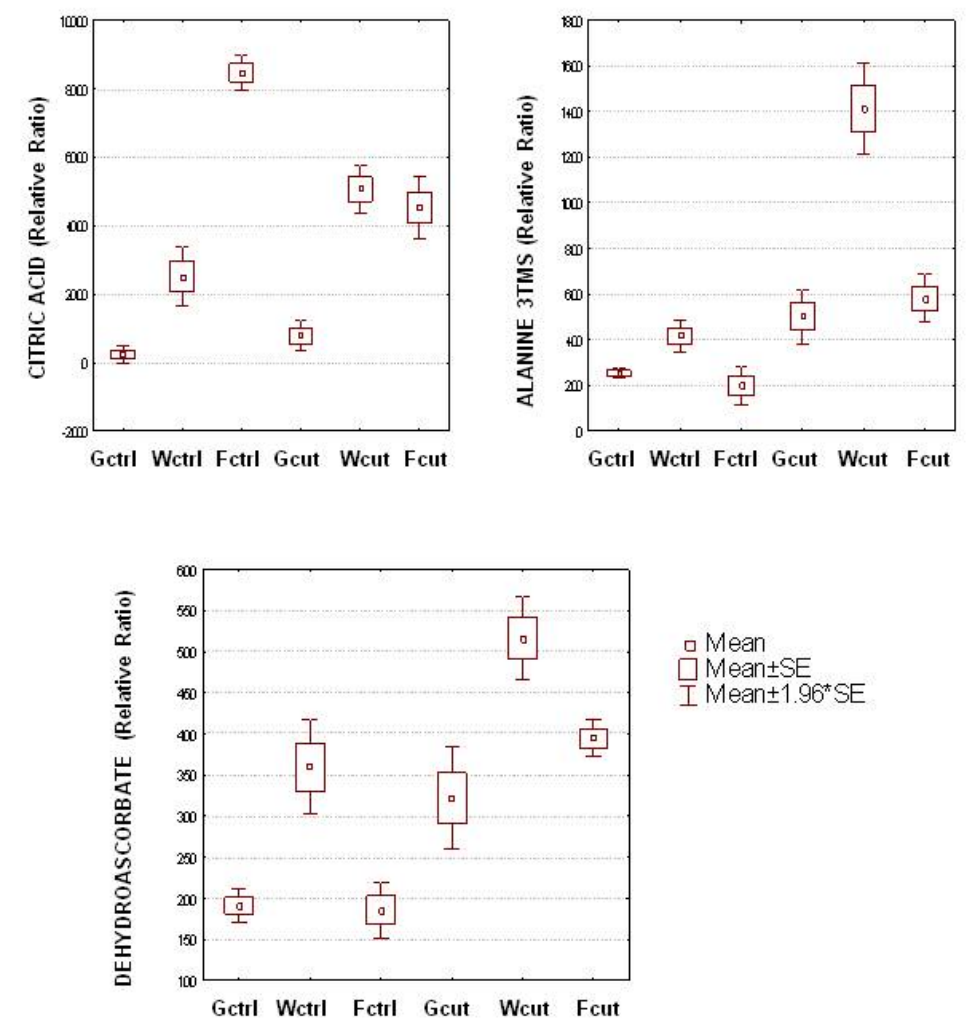

- Mean

I Mean $\pm 1.96 * \mathrm{SE}$

Fig. 3. A) Significantly regulated organic acids in the three different types of peach fruits $24 \mathrm{~h}\left(\mathrm{~T}_{1}\right)$ after cutting in comparison to fruits at the same stage with no cutting (control). G='Ghiaccio3', W='Wilhelmina', $\mathrm{F}=$ ='Fayette'. B) Four metabolites that showed significant changes after cutting in the three peach cultivars. Relative ratio between area peaks were indicated (Compound/Internal standard). Gctrl='Ghiaccio3' control, Gcut='Ghiaccio3' after cutting, Fctrl='Fayette' control, Fcut='Fayette' after cutting, Wctrl='Wilhelmina' control, Wcut='Wilhelmina' after cutting. 
1022 